ARTIGO ORIGINAL

ISSN 1677-5090

(C) 2019 Revista de Ciências Médicas e Biológicas

http://dx.doi.org/10.9771/cmbio.v18i3.34179

\title{
Tipos craniofaciais e relação com a síndrome da apneia obstrutiva do sono
}

\author{
Craniofacial types and relation to obstructive sleep apnea syndrome
}

\begin{abstract}
Ranuzia Mercês Santos Galtieri' ${ }^{1}$ Cristina Salles², Ailton Melo³ ${ }^{3}$ Adelmir Souza-Machado**
${ }^{1}$ Odontológa. Mestranda do Programa de Pós-Graduação em Processos Interativos dos Órgãos e Sistemas, Instituto de Ciências da Saúde, Universidade Federal da Bahia. ${ }^{2}$ Doutora em Ciências da Saúde pela Universidade Federal da Bahia. Docente da Escola Baiana de Medicina e Saúde Pública. ${ }^{3}$ Doutor em Ciências Biológicas pela Universidade Federal de São Paulo. Docente da Faculdade de Medicina da Bahia pela Universidade Federal da Bahia. ${ }^{4}$ Doutor em Medicina e Saúde pela Universidade Federal da Bahia. Docente do Programa de Pós-Graduação em Processos Interativos dos Órgãos e Sistemas, Universidade Federal da Bahia.
\end{abstract}

\begin{abstract}
Resumo
Introdução: fatores anatômicos, como obesidade e alterações craniofaciais, interagem na etiologia e na expressão da Síndrome da Apneia Obstrutiva do Sono. Dimensões cranianas e faciais são usadas para classificar os diferentes fenótipos mediante duas medidas antropométricas: o índice craniano e o índice facial. Objetivo: avaliar a possível associação dos tipos cranianos e faciais ao risco da Síndrome da Apneia Obstrutiva do Sono, obtido pelo questionário de Berlim. Metodologia: participaram do estudo 34 indivíduos (76,5\% mulheres), com mediana de idade $=59,0(56,8-65,0)$ anos, avaliados quanto ao risco da Síndrome da Apneia Obstrutiva do Sono, através do questionário de Berlim e medidas antropométricas: índice de massa corporal, circunferência cervical, circunferência abdominal, índice craniano e índice facial. Resultados: foram encontradas as medidas índice de massa corporal = 30,3 $(24,7-37,4)$ $\mathrm{kg} / \mathrm{m}^{2}$, circunferência cervical $=37,6(33,9-41,6) \mathrm{cm}$ e circunferência abdominal $=102,0(91,8-116,1) \mathrm{cm}$. No grupo de alto risco para essa Síndrome, houve predominância do tipo craniano braquicefálico $=40,7 \%$, e do tipo facial euriprosópico $=37,0 \%$. Conclusão: através do presente estudo foi possível observar que idade, obesidade, tipo craniano braquicefálico e face euriprosópica associaram-se ao alto risco de Síndrome da Apneia Obstrutiva do Sono.
\end{abstract}

Palavras-chave: Sono. Síndromes da Apneia do Sono. Ronco. Antropometria. Crânio. Face.

\begin{abstract}
Introduction: anatomical factors such as obesity and craniofacial alterations interact in the etiology and expression of Obstructive Sleep Apnea Syndrome. Cranial and facial dimensions are used to classify the different phenotypes through two anthropometric measurements: the cranial and facial indexes. Objective: to evaluate the possible association of cranial and facial types with Obstructive Sleep Apnea Syndrome risk obtained by the Berlin questionnaire. Methodology: the study included thirty-four individuals (76,5\% women), with a median age $=59,0(56,8-65,0)$ years, who were evaluated for Obstructive Sleep Apnea Syndrome risk through the Berlin questionnaire and anthropometric measurements: body mass index, cervical circumference, abdominal circumference, cranial index and facial index. Results: the following measurements were found: body mass index $=30,3(24,7-37,4) \mathrm{kg} / \mathrm{m}^{2}$, cervical circumference $=37,6(33,9-41,6) \mathrm{cm}$ and abdominal circumference $=102,0(91,8-116,1) \mathrm{cm}$. In the high-risk group for this Syndrome there was a predominance of brachycephalic cranial type $=40,7 \%$, and euryprosopic facial type $=37,0 \%$. Conclusion: through the present study, it was possible to observe that age, obesity, brachycephalic cranial type and euryprosopic face were associated with the high-risk for Obstructive Sleep Apnea Syndrome.
\end{abstract}

Keywords: Sleep. Sleep Apnea Syndromes. Snoring. Anthropometry. Skull. Face.

\section{INTRODUÇÃO}

A Síndrome da Apneia Obstrutiva do Sono (SAOS) é um distúrbio respiratório do sono (DRS), caracterizado por episódios recorrentes de completa ou parcial obstrução do fluxo aéreo, associado com dessaturação de oxihemoglobina e aumento do esforço respiratório (AMERICAN ACADEMY OF SLEEP MEDICINE, 1999; GUILLEMINAULT;

Correspondente/Corresponding: *Adelmir Souza-Machado - Instituto de Ciências da Saúde - Universidade Federal da Bahia - End: Av. Reitor Miguel Calmon s/n - Vale do Canela. CEP: 40231-300. Salvador-BA. Tel : (71) 98875-9572 — E-mail: adelmirm@gmail.com.
TILKIAN; DEMENT, 1976). O quadro clínico da SAOS caracteriza-se por ronco, sonolência excessiva diurna, pausas respiratórias durante o sono, podendo ocorrer prejuízo das funções cognitivas, como concentração, atenção, memória (BITTENCOURT et al., 2009) e alterações de humor, como ansiedade, irritabilidade e depressão (OSMAN et al., 2018). A SAOS está relacionada com complicações cardiocirculatórias (GOZAL; KHEIRANDISH-GOZAL, 2008), acidentes automobilísticos (KOCHUPURACKAL; MATHEW; WT, 2019) e aumento da mortalidade (MARIN-OTO; VICENTE; MARIN, 2019). Nos Estados Unidos, a prevalência da SAOS na população de meia idade mostrou-se em torno 
de $4 \%$ para os homens e $2 \%$ para as mulheres (YOUNG et al., 1993). Em 2018, na cidade de São Paulo, dados epidemiológicos revelaram a prevalência da SAOS em $32,9 \%$ da população adulta, atingindo $26,1 \%$ das mulheres e $40,6 \%$ dos homens examinados (TUFIK et al., 2010).

A etiologia da SAOS envolve fatores genéticos, anatômicos, hormonais e neuromusculares os quais interagem entre si (SUBRAMANI et al., 2016). Dentre os fatores anatômicos, incluem-se obesidade, excesso de tecido mole nas vias aéreas superiores (VAS) e alterações craniofaciais (VEASEY; ROSEN, 2019). A cefalometria é o método mais utilizado para analisar as características craniofaciais e sua relação com SAOS (SALLES et al., 2005), contudo é um exame que apresenta limitações na avaliação do tamanho e forma do crânio, os quais estão diretamente relacionados com as variações anatômicas da face (ENLOW et al., 1971) e das VAS, refletindo a maior chance de colapso dessas estruturas (CAKIRER et al., 2001). Dessa maneira, tem sido considerado utilizar as medidas antropométricas craniofaciais como uma das ferramentas clínicas para identificação dos tipos cranianos e faciais em indivíduos que apresentam risco para SAOS.

Dessa forma, o presente estudo tem como objetivo avaliar a associação dos tipos craniofaciais com o risco de SAOS, obtido pelo questionário de Berlim, nos indivíduos atendidos no Serviço de Referência em Medicina do Sono, do Hospital Universitário Professor Edgar Santos (HUPES), Universidade Federal da Bahia (UFBA), Salvador, Bahia.

\section{METODOLOGIA}

Trata-se de um estudo do tipo corte transversal, realizado no Serviço de Referência em Medicina do Sono, no Hospital Universitário Professor Edgar Santos, localizado em Salvador, Bahia. O estudo foi aprovado pelo Comitê de Ética em Pesquisa do referido Hospital, conforme Parecer consubstanciado $\mathrm{n}$ - 2.962.523.

Foram avaliados 34 indivíduos, durante o período janeiro a julho de 2019, com os seguintes critérios de inclusão; adultos entre 18 a 70 anos, ambos os sexos, presença de queixa de ronco, apneia do sono referida e sonolência excessiva diurna. Foram excluídos os indivíduos com doença ou comprometimento que impediria a realização de questionários, diagnóstico de tumor em vias aéreas superiores e história prévia de cirurgia ortognática.

Todos os pacientes estavam matriculados no Serviço de Referência de Medicina do Sono e durante a consulta, para investigação diagnóstica com o médico especialista, foram informados e selecionados para a pesquisa. Somente participaram aqueles que assinaram o Termo de Consentimento Livre e Esclarecido (TCLE), em consonância com os aspectos éticos previstos na Resolução no 466/12, do Conselho Nacional de Saúde, respeitando-se os princípios da declaração de Helsinque. Após o atendimento médico, todos os pacientes foram submetidos a um protocolo de avaliação, por uma pesquisadora, que incluiu a aplicação do questionário de Berlim e medidas antropométricas: peso, altura, índice de massa corporal (IMC), circunferência cervical (CC), circunferência abdominal (CA) e índice craniano (IC) e índice facial (IF).

Para avaliação do risco de SAOS, adotou-se o questionário de Berlim já validado para a língua portuguesa, o qual oferece efetividade na triagem quando há suspeita de SAOS. O questionário demonstrou valores de sensibilidade de $69 \%$ a $86 \%$ e especificidade de 56 a $95 \%$, valor preditivo positivo de $77 \%$ a $96 \%$, para detecção de SAOS (ABRISHAMI; KHAJEHDEHI; CHUNG, 2010), discriminando indivíduos com baixa e alta chance de desenvolver SAOS. Ele é constituído de três categorias, sendo que a primeira inclui questões sobre a presença do ronco, a sua intensidade e frequência, o incômodo do ronco a terceiros e o relato de apneias presenciadas; na segunda, questionam-se os episódios de sonolência ao acordar, ao longo do dia e também na direção de veículos; por fim, na última categoria, lista-se a história de hipertensão arterial sistêmica (HAS) e calcula-se o IMC. A positividade em duas ou três categorias define um score de alto risco para SAOS e a presença de apenas um domínio positivo ou nenhum resulta em um score de baixo risco. (ARAÚJO-MELO et al., 2016; OLIVEIRA et al., 2013).

$O$ peso foi mensurado em uma balança mecânica (Filizola ${ }^{\circledR}$ ), a altura foi medida com um estadiômetro e o IMC foi calculado através da fórmula: peso por quilogramas dividido pela altura em metros ao quadrado. O IMC foi classificado de acordo com definição da Organização Mundial de Saúde da seguinte forma: normal (18,5 a 24,9 $\left.\mathrm{kg} / \mathrm{m}^{2}\right)$; sobrepeso $\left(25,0\right.$ a $\left.29,9 \mathrm{~kg} / \mathrm{m}^{2}\right)$; obesidade grau I (30,0 a $\left.34,9 \mathrm{~kg} / \mathrm{m}^{2}\right)$; obesidade grau II (35,0 a $39,9 \mathrm{~kg}$ / $\mathrm{m}^{2}$ ) e obesidade grau III (> e igual a $40,0 \mathrm{~kg} / \mathrm{m}^{2}$ ) (WORLD HEALTH ORGANIZATION, 1997).

A circunferência cervical foi medida com o indivíduo sentado, utilizando-se uma fita métrica flexível e inextensível, no nível da cartilagem crico-tireoídea, em uma linha perpendicular ao longo eixo do pescoço, com o paciente em apneia inspiratória e com a cabeça paralela ao solo (PINTO et al., 2011). Para a aferição da circunferência abdominal, os indivíduos foram medidos em posição ortostática, com os braços afastados, pés juntos e abdome relaxado no final do movimento expiratório. Foi utilizada a mesma fita métrica, posicionada em nível de um ponto médio localizado entre a crista ilíaca superior e a última costela (PIMENTA et al., 2014).

Para avaliação das medidas craniofaciais, realizadas por uma única pesquisadora, com compasso antropométrico, o paciente se posicionou sentado, com plano de Camper (plano que vai do trágus à asa do nariz), paralelo ao solo e a cabeça em posição neutra. $O$ índice craniano foi definido como a razão entre largura máxima craniana e comprimento máximo craniano (Figura 1A). Largura máxima craniana é o maior diâmetro transversal da cabeça e comprimento máximo craniano é a distância entre o ponto mais inferior abaixo da glabela (o ponto mais proeminente, no plano mediano, entre as sobrancelhas) e o ponto de projeção mais distante no plano sagital mediano, 
na parte de trás da cabeça (opistocrânio). $O$ índice facial foi descrito como a razão entre a altura násio-gnátio e a largura bizigomática (Figura 1B). Altura násio-gnátio é a distância entre o ponto násio (local em que uma tangente horizontal que passa nos pontos mais altos dos sulcos palpebrais superiores intercepta o plano sagital mediano) e o gnátio é o ponto médio mais inferior no bordo da mandíbula. Largura bizigomática é a distância entre os pontos situados mais lateralmente entre os arcos zigomáticos de ambos os lados. (FARKAS; POSNICK; HRECZKO, 1992; MONTAGU; BROZEK, 1960).

Figura 1A - Índice Craniano

Índice Craniano $=$ Largura máxima craniana $\times 100$ Comprimento máximo craniano

Figura 1B - Índice Facial

Índice Facial $=\quad$ Altura násio-gnátio $\times 100$ Largura bizigomática

Fonte: Montagu e Brozek (1960).

A classificação do tipo craniano é estabelecida de acordo com o índice craniano e representada da seguinte forma: ultradolicocefálico (IC < 64,9), hiperdolicocefálico (IC = 65,0 - 69,9), dolicocefálico (IC = 70,0 - 74,9), mesocefálico (IC = 75,0 - 79,9), braquicefálico (IC = 80,0 - 84,9), hiperbraquicefálico (IC $=85,0-89,9)$ e ultrabraquicefálico (IC > 90,0). Da mesma forma, a classificação do tipo facial é estabelecida de acordo com o índice facial e assim representada: hipereuriprosópico (IF < 79,9), euriprosópico (IF $=80,0-84,9)$, mesoprosópico (IF = 85,0 - 89,9), leptoprosópico (IF $=90,0-94,9$ ) e hiperleptoprosópico (IF > 95,0) (FRANCO; ARAÚJO; VOGEL; QUINTÃO, 2013).

$\mathrm{Na}$ classificação craniana existem dois tipos extremos: de um lado, o braquicefálico e do outro o dolicocefálico. $O$ tipo braquicefálico apresenta a maior largura biparietal e uma dimensão occipital-frontal mais curta.
Indivíduos dolicocefálicos exibem uma distância biparietal mais estreita e um maior comprimento anteroposterior. Os tipos faciais apresentam seus extremos; euriprosópicos e leptoprosópicos. O tipo euriprosópico apresenta a face mais larga do sentido bizigomático e mais curta na dimensão násio-gnátio (vertical). Em contrapartida, o indivíduo leptoprosópico tem maior comprimento vertical e menor dimensão bizigomática. Pode ocorrer também um tipo intermediário, mesocefálico, em que a forma de cabeça e as feições faciais tendem a ser correspondentemente intermediárias, mesoprosópicas (ENLOW, 1993).

Para construção do banco de dados e cálculos estatísticos, foi utilizado o programa estatístico Statistical Package for the Social Sciences (SPSS). O resultado das variáveis foi apresentado sob a forma de mediana e amplitude interquartil e a comparação entre os grupos foi feita através do teste de Mann-Whitney.

\section{RESULTADOS}

As características gerais e antropométricas da amostra estudada, assim como os valores comparativos entre os homens e mulheres, estão representadas na Tabela 1. $\mathrm{Na}$ avaliação da frequência dos tipos cranianos da amostra, observou-se que o tipo braquicefálico demonstrou maior percentagem, $38,2 \%$, seguido do tipos mesocefálico, $26,5 \%$, hiperbraquicefálico, $23,5 \%$, ultrabraquicefálico e dolicocefálico, 5,9\%. No grupo masculino, a característica braquicefálica foi predominante, $62,5 \%$, ao passo que no grupo feminino apresentaram valores semelhantes quantos aos tipos braquicefálicos e mesocefálicos. Em relação ao índice facial, tanto homens como mulheres apresentaram características euriprosópicas. A frequência dos tipos faciais da amostra revelou o maior percentual para os tipos euriprosópicos $32,4 \%$. O percentual dos tipos hipereuriprosópicos e mesoprosópicos apresentaram valores semelhantes (29,4\% para cada tipo), enquanto a frequência para os tipos leptoprosópicos e hiperleptoprosópicos representaram $5,9 \%$ e 2,9\%, respectivamente.

Tabela 1 - Características gerais e antropométricas da amostra estudada e de acordo com o gênero.

\begin{tabular}{lrrr}
\hline & \multicolumn{1}{c}{ Total $\mathbf{( N = 3 4 )}$} & \multicolumn{1}{c}{$\mathbf{H}(\mathbf{N}=\mathbf{8})$} & \multicolumn{1}{c}{$\mathbf{M}$ (N = 26) } \\
\hline Idade (anos) & $59,0(56,8-65,0)$ & $58,0(55,5-59,0)$ & $61,0(56,8-65,0)$ \\
IMC, kg/m² & $30,3(24,7-37,4)$ & $30,3(23,5-35,1)$ & $30,6(25,7-38,5)$ \\
CC (cm) & $37,6(33,9-41,6)$ & $41,0(37,9-45,8)$ & $36,6(33,0-40,3)$ \\
CA (cm) & $102,0(91,8-116,1)$ & $107,5(93,3-116,4)$ & $102,0(90,8-116,5)$ \\
LMC (cm) & $15,0(14,5-16,0)$ & $16,0(14,6-16,3)$ & $15,0(14,5-15,5)$ \\
CMC (cm) & $18,5(18,0-19,0)$ & $18,5(18,3-19,7)$ & $18,5(17,9-19,0)$ \\
IC & $81,7(78,7-85,4)$ & $82,6(80,0-85,6)$ & $81,6(79,0-85,4)$ \\
ANG (cm) & $11,5(10,9-12,0)$ & $11,9(10,8-12,6)$ & $11,1(10,8-11,6)$ \\
LBZ (cm) & $13,5(13,0-14,1)$ & $14,2(13,9-14,7)$ & $13,3(12,8-13,6)$ \\
IF & $82,9(78,2-88,0)$ & $81,9(77,9-86,7)$ & $84,0(78,2-88,2)$ \\
\hline
\end{tabular}

Fonte: Dados da pesquisa.

Legenda: $N=$ número de sujeitos; $H=$ homens; $M=$ mulheres; $I M C=$ índice de massa corporal; $C C=$ circunferência cervical; $C A=$ circunferência 
abdominal; $\mathrm{cm}=$ centímetros; $\mathrm{kg}=$ kilograma; $\mathrm{m}^{2}=$ metro ao quadrado; $L M C=$ Largura máxima craniana; $C M C=$ Comprimento máximo craniano; $I C=$ Índice craniano; $A N G=$ Altura násio-gnátio; $L B Z=$ Largura bizigomática; IF = Índice facial .

As características gerais e antropométricas da amos- de baixo risco para SAOS, houve predominância do tipo tra estudada, de acordo com o risco para SAOS, estão representadas na Tabela 2. Na amostra estudada, foi observado que 7 indivíduos $(20,6 \%)$ apresentaram baixo risco e 27 (79,4\%), alta probabilidade para SAOS. No grupo craniano mesocefálico, $42,9 \%$, e do tipo facial mesoprosópico, 42,9\%. Por outro lado, no grupo de alto risco, a maior frequência foi do tipo craniano braquicefálico, $40,7 \%$, e o tipo facial euriprosópico, $37,0 \%$.

Tabela 2 - Características gerais e antropométricas da amostra estudada e de acordo com o risco para SAOS.

\begin{tabular}{lccc}
\hline & \multicolumn{1}{c}{$\begin{array}{c}\text { Total } \\
(\mathbf{N}=\mathbf{3 4})\end{array}$} & $\begin{array}{c}\text { Baixo risco } \\
\mathbf{( N = 7 )}\end{array}$ & $\begin{array}{c}\text { Alto risco } \\
(\mathbf{N}=\mathbf{2 7})\end{array}$ \\
\hline Idade(anos) & $59,0(56,8-65,0)$ & $61,0(59,0-66,0)$ & $59,0(56,0-65,0)$ \\
IMC, kg/m² & $30,3(24,7-37,4)$ & $26,0(22,1-37,3)$ & $31,2(26,8-37,9)$ \\
CC (cm) & $37,6(33,9-41,6)$ & $35,0(33,5-41,0)$ & $38,0(35,0-42,0)$ \\
CA (cm) & $102,0(91,8-116,1)$ & $88,0(80,0-122,5)$ & $106,0(97,0-116,0)$ \\
LMC (cm) & $15,0(14,5-16,0)$ & $15,3(14,4-15,5)$ & $15,0(14,6-16,0)$ \\
CMC (cm) & $18,5(18,0-19,0)$ & $18,0(17,8-19,0)$ & $18,5(18,1-19,0)$ \\
IC & $81,7(78,7-85,4)$ & $80,0(78,8-85,6)$ & $81,8(78,4-85,4)$ \\
ANG (cm) & $11,5(10,9-12,0)$ & $11,5(11,0-12,0)$ & $11,2(10,6-12,0)$ \\
LBZ (cm) & $13,5(13,0-14,1)$ & $13,5(13,2-14,2)$ & $13,3(12,9-14,1)$ \\
IF & $82,9(78,2-88,0)$ & $85,2(77,0-88,8)$ & $82,1(78,5-88,0)$ \\
\hline
\end{tabular}

Fonte: Dados da pesquisa.

Legenda: $N=$ número de sujeitos; $I M C$ = índice de massa corporal; $C C$ = circunferência cervical; $C A$ = circunferência abdominal; $\mathrm{cm}=$ centímetros; $\mathrm{kg}=$ kilograma; $\mathrm{m}^{2}=$ metro ao quadrado; $L M C=$ Largura máxima craniana; $C M C=$ Comprimento máximo craniano; $I C=$ Índice craniano; $A N G=$ Altura násio-gnátio; $L B Z=$ Largura bizigomática; IF = Índice facial.

\section{DISCUSSÃO}

No presente estudo, observou-se que a maioria dos indivíduos que apresentaram alto risco para SAOS têm características braquicefálicas. Sutherland et al. (2012) também descreveram que a morfologia craniofacial é importante fator de risco para SAOS. Cakirer et al. (2001), ao estudarem caucasianos apneicos através de duas medidas antropométricas (índice craniano e índice facial), encontraram maior prevalência do padrão de crescimento braquicefálico. Redline et al. (1997) compararam indivíduos caucasianos e afro-americanos apneicos e não apneicos através de questionários, polissonografia, cefalometria, medidas de tecido mole e antropométricas. A cefalometria e o índice craniano mostraram diferenças quanto às medidas e posições das estruturas de tecido mole e ósseas entre os grupos. Ao compararem apneicos caucasianos e afro-americanos, observaram prevalência do tipo braquicefálico em $36 \%$ dos caucasianos e $17 \%$ dos afro-americanos. Indivíduos que tendem a braquicefalia exibem redução anteroposterior da base craniana, maior flexão da base do crânio, redução da fossa nasal e redução anteroposterior das vias aéreas (REDLINE et al., 1997).

A predominância do tipo facial euriprosópico, no atual estudo, associou-se ao tipo craniano braquicefá- lico, no qual os indivíduos têm a forma facial mais larga e mais curta (ENLOW, 1993). Esses resultados estão em consonância com Cleveland Family Study, onde a média do índice facial foi de $84,5 \pm 6,1$, em 74 apneicos. Capistrano et al. (2015) associaram os padrões morfológicos e tipos faciais com diagnóstico de SAOS em 252 pacientes, através de fotografias faciais de frente, perfil e sorriso. $O$ diagnóstico do tipo facial foi estabelecido por meio de um índice facial que leva em consideração a proporção entre a largura e altura da face. 0 desenho morfológico facial mostrou que indivíduos com características euriprosópicas apresentaram IAH mais elevados.

No atual estudo, houve predominância da quinta a sexta década de vida, semelhante aos achados do Wisconsin Sleep Cohort Study, composto por 1520 indivíduos, tendo maior prevalência para a SAOS a faixa etária acima dos 60 anos (PEPPARD et al., 2013). No Brasil, estudo epidemiológico na cidade de São Paulo que incluiu 1042 pessoas, Tufik et al. (2010) encontram a frequência de SAOS para a faixa etária de 50 a 60 anos, de 49,2\% (55,9\% para homens e $43,9 \%$ para mulheres), na de 60 a 70 anos, de $60,2 \%$ (55,9\% para homens e $63,4 \%$ para mulheres) e na de 70 a 80 anos, de $86,9 \%$ ( $88,7 \%$ para homens e $85,8 \%$ para mulheres). 
No atual estudo, o sexo feminino apresentou maior frequência de alto risco da SAOS. No entanto, de acordo com Lin et al. (2008), a prevalência da SAOS é maior em homens do que em mulheres. Estima-se que a proporção entre homens e mulheres seja de 3: 1 a 5: 1 na população geral e, 8: 1 a 10: 1 em populações com distúrbios do sono. Os níveis elevados de andrógenos podem atuar relaxando os músculos dilatadores da faringe, favorecendo o colapso das VAS, e alterar a composição da gordura corpórea aumentando o depósito de tecido mole na faringe (FOGEL et al., 2001). Nos adultos jovens há maior prevalência da SAOS em homens, mas após a menopausa a prevalência nas mulheres aumenta. A menopausa tem sido identificada como um fator de risco para a SAOS, sugerindo que os hormônios sexuais femininos, particularmente a progesterona, protejam as mulheres da SAOS, influenciando a atividade dilatadora dos músculos das vias aéreas (MARTINS et al., 2007). Os fatores de risco, idade avançada, gênero masculino e menopausa associam-se à prevalência de distúrbios respiratórios do sono (DALTRO et al., 2006).

O presente estudo corrobora a literatura, ao demonstrar que o IMC da amostra foi caracterizado por obesidade grau 1 . O IMC superior a $30 \mathrm{~kg} / \mathrm{m}^{2}$ é sugestivo de SAOS, particularmente quando a deposição de gordura assume padrão central, caracterizado pelo aumento do perímetro cervical $(>43 \mathrm{~cm}$ nos homens e $>38 \mathrm{~cm}$ nas mulheres) e/ou da circunferência abdominal ( $>102 \mathrm{~cm}$ em homens e $>88 \mathrm{~cm}$ nas mulheres) (DRAGER et al., 2018). A circunferência do pescoço é um fator predisponente para SAOS, pois o excesso de adiposidade nessa região leva ao estreitamento das VAS (POLESEL et al., 2019). Existe uma correlação positiva entre idade, obesidade, circunferência de pescoço e circunferência abdominal, favorecendo o risco para SAOS (MARTIN et al., 1997).

No presente estudo, foi possível observar que a idade, obesidade, tipo craniano braquicefálico e face euriprosópica associaram-se ao alto risco de SAOS.

\section{REFERÊNCIAS}

ABRISHAMI, A.; KHAJEHDEHI, A.; CHUNG, F. A systematic review of screening questionnaires for obstructive sleep apnea. Can. J. Anesth., Canadá, v. 57, n. 5, p. 423-438, 2010.

AMERICAN ACADEMY OF SLEEP MEDICINE TASK FORCE. Sleep-related breathing disorders in adults: recommendations for syndrome definition and measurement techniques in clinical research. Sleep, New York, v. 22, n. 5, p. 667-689, 1999.

ARAÚJO-MELO, M. H. et al. Questionários e escalas úteis na pesquisa da síndrome da apneia obstrutiva do sono. Revista HUPE, Rio de Janeiro, v.15, n.1, p. 49-55, jan./mar. 2016.

BITTENCOURT, L. R. A. et al. Abordagem geral do paciente com síndrome da apneia obstrutiva do sono. Rev. Bras. Hipertens., Rio de Janeiro, v.16, n.3, p.158-163, 2009.

CAKIRER, B. et al. The relationship between craniofacial morphology and obstructive sleep apnea in whites and in African-Americans. Am. J. Respir. Crit. Care Med., New York, v. 163, n. 4, p. 947-950, 2001.

CAPISTRANO, A. et al. Facial morphology and obstructive sleep apnea.
Dental Press J. Orthod., Maringá, v. 20, n. 6, p. 60-67, Nov./Dec. 2015.

DALTRO, C. H. da C. et al. Síndrome da apnéia e hipopnéia obstrutiva do sono: associação com obesidade, gênero e idade. Arq. Bras. Endocrinol. Metab., São Paulo, v. 50, n.1, p. 74-81, fev. 2006.

DRAGER, L. F. et al.10 Posicionamento brasileiro sobre o impacto dos distúrbios de sono nas doenças cardiovasculares da Sociedade Brasileira de Cardiologia. Arq. Bras. Cardiol., São Paulo, v.111, n. 2, p. 290-340, ago. 2018.

ENLOW, D. Faces. In: ENLOW, D. Crescimento facial. 3. ed. Porto Alegre: Artes Médicas, 1993. p. 1-6. cap. 1.

ENLOW, D. H.; KURODA, T.; LEWIS, A. B. The morphological and morphogenetic basis for craniofacial form and pattern. Angle Orthod., Appleton, v. 41, n. 3, p. 161-188, 1971.

FARKAS, L.G.; POSNICK, J.C.; HRECZKO, T. M. Growth patterns of the face: a morphometric study. Cleft Palate-Craniofac., J., Lewiston, v. 29, n. 4, p. 308-315, July 1992.

FOGEL, R. B. et al. Increased prevalence of obstructive sleep apnea syndrome in obese women with polycystic ovary syndrome. J. Clin. Endocrinol. Metab., Philadelphia, v. 86, n. 3, p.1175-1180, 2001.

FRANCO, F. C. M. et al. Brachycephalic, dolichocephalic and mesocephalic: is it appropriate to describe the face using skull patterns? Dental Press J. Orthod., Maringá, v.18, 3, p.159-163, May/Jun. 2013.

GOZAL, M. D.; KHEIRANDISH-GOZAL, L. Cardiovascular morbidity in obstructive sleep apnea oxidative stress, inflammation, and much more. Am J. Respir. Crit. Care Med., New York, v. 177, n. 4, p. 369-375, 2008.

GUILLEMINAULT, C.; TILKIAN, A.; DEMENT, W. C. The sleep apnea syndromes. Annu Rev Med., London, v. 27, p.465-484, 1976.

KOCHUPURACKAL, M. M.; MATHEW, R. R.; WT, D. A review on obstructive sleep apnea. Int. J. Curr. Res., India, v. 11, n. 1, p. 767-770, Jan. 2019.

LIN, C. M.; DAVIDSON, T. M.; ANCOLI-ISRAEL, S. Gender differences in obstructive sleep apnea and treatment implications. Sleep Med. Rev., London, v.12, n. 6, p. 481-496, Dec. 2008.

MARIN-OTO, M.; VICENTE, E. E.; MARIN, J. M. Long term management of obstructive sleep apnea and its comorbidities. Multidiscip. Respir. Med., London, v.14, n. 21, p. 1-9, 2019.

MARTINS, A.B.; TUFIK, S.; MOURA, S. M. G. P. T. Síndrome da apnéia-hipopnéia obstrutiva do sono. Fisiopatologia. J. Bras. Pneumolog., Brasília, v. 33, n. 1, p. 36-43, 2007.

MARTIN, S. E. et al. The effect of age, sex, obesity and posture on upper airway size. Eur. Respir. J., Copenhagen, v.10, n 9, p. 2087-2090, 1997.

MONTAGU, M. F.; BROSEK, J. Craniometric Indices. In: MONTAGU, M. F. A handbook of anthropometry. Chicago: Charles C. Thomas, 1960.

OLIVEIRA, H. D. et al. Características orofaciais e risco para apneia obstrutiva do sono em hipertensos. R. Enferm. Cent. O. Min., Itaúna, MG, v. 3, n. 2, p.706-713, mai/ago. 2013.

OSMAN, A. M. et al. Obstructive sleep apnea: current perspectives. Nat. Sci. Sleep., v. 10, p. 21-34, 2018.

PEPPARD, P. E. et al. Increased prevalence of sleep-disordered breathing in adults. Am. J. Epidemiol., Baltimore, v. 177, n. 9, p 1006-1014, Apr. 2013.

PIMENTA, I. L. et al. Medidas das circunferências abdominal e cervical para mensurar riscos cardiovasculares. Rev. Med. Minas Gerais, Belo Horizonte, v. 24, supl 9, p. S16-S19, 2014. 
PINTO, J. A. et al. Antropometric data as predictors of obstructive sleep apnea severity. Braz. J. Otorhinolaryngol., São Paulo, v. 77, n. 4, p. 516521, jul/ago, 2011.

POLESEL, D. N. et al. Gender differences in the application of anthropometric measures for evaluation of obstructive sleep apnea. Sleep Sci., São Paulo, v.12, n.1, p. 2-9, 2019.

REDLINE, S. et al. Racial differences in sleep-disordered breathing in African-Americans and Caucasians. Am. J. Respir. Crit. Care Med., New York, v. 155, p. 186-192, 1997.

SALLES, C. et al. Síndrome da ápneia e hipopnéia do sono: análise cefalométrica. Rev. Bras. Otorrinolarigol., São Paulo, v. 71, n. 3, p. 369-372, maio/jul. 2005.

SUBRAMANI, Y. et al. Understanding phenotypes of obstructive sleep apnea: applications in anesthesia, surgery, and perioperative medicine.
Anesth. Analg., Cleveland, v.124, n.1, p.179-191, Jan, 2017.

SUTHERLAND, K.; LEE, R. W. W.; CISTULLI, P. A. Obesity and craniofacial structure as risk factors for obstructive sleep apnoea: impact of ethnicity. Respirol., Carlton, v. 17, p. 213-222, 2012.

TUFIK, S. et al. Obstrutive sleep apnea syndrome in the São Paulo epidemiologic sleep study. Sleep Medicine, Amsterdam, v.11, n. 5, p. 441-446, May, 2010.

VEASEY, S. C.; ROSEN, I. M. Obstructive sleep apnea in adults. N. Engl. J. Med., Boston, v. 380, n.15, p. 1442-1449, Apr. 2019.

WORLD HEALTH ORGANIZATION. Obesity: Preventing and managing the global epidemic. Geneva: 1997.

YOUNG, T. et al. The occurrence of sleep disordered-breathing among middle aged adults. N. Engl. J. Med., Boston, v. 328, n.17, p. $1230-$ 1239, 1993.

Submetido em: 04/11/2019

Aceito em: 30/11/2019 